\title{
Relationship between genetic polymorphisms in the DRD5 gene and paranoid schizophrenia in northern Han Chinese
}

\author{
Y. Zhao, M. Ding, H. Pang, X.M. Xu and B.J. Wang \\ Department of Forensic Medicine, China Medical University, \\ Shenyang, China \\ Corresponding author: B.J. Wang \\ E-mail: bjwang@mail.cmu.edu.cn \\ Genet. Mol. Res. 13 (1): 1609-1618 (2014) \\ Received May 20, 2013 \\ Accepted October 15, 2013 \\ Published March 12, 2014 \\ DOI http://dx.doi.org/10.4238/2014.March.12.13
}

\begin{abstract}
Dopamine (DA) has been implicated in the pathophysiology of several psychiatric disorders, including schizophrenia. Thus, genes related to the dopaminergic (DAergic) system are good candidate genes for schizophrenia. One of receptors of the DA receptor system is dopamine receptor 5 (DRD5). Single nucleotide polymorphisms (SNPs) in the regulatory regions of $D R D 5$ gene may affect gene expression, influence biosynthesis of DA and underlie various neuropsychiatric disorders related to DA dysfunction. The present study explored the association of SNPs within the DRD5 gene with paranoid schizophrenia in Han Chinese. A total of 176 patients with schizophrenia and 206 healthy controls were genotyped for four DRD5 SNPs (rs77434921, rs2076907, rs6283, and rs1800762). Significant group differences were observed in the allele and genotype frequencies of rs77434921 and rs1800762 and in the frequencies of GC haplotypes corresponding to rs77434921-rs1800762. Our findings suggest that common genetic variations of DRD5 are likely to contribute to genetic susceptibility to paranoid schizophrenia in Han Chinese. Further studies in larger samples are needed to replicate this association.
\end{abstract}

Key words: DRD5; Dopamine; Paranoid schizophrenia; Haplotype; SNP 


\section{INTRODUCTION}

Schizophrenia is a complex disorder affecting approximately $1 \%$ of the population worldwide (Schwab and Wildenauer, 2008), with the paranoid type being the most common. The etiology of schizophrenia remains unclear. High heritability $(\sim 80 \%)$ and higher concordance in monozygotic $(\sim 50 \%)$ than in dizygotic $(\sim 17 \%)$ twins are strong indicators for an inherited basis of schizophrenia (Cardno and Gottesman, 2000; Pal et al., 2009). Recent advances in human genome information and molecular biology techniques have facilitated significant progress in genetic studies of schizophrenia. A number of important candidate genes have been cloned and their preliminary positions identified. These genes are closely related to function, and thus have revealed broad contours of disease-related signaling pathways.

DA is a neurotransmitter synthesized in the central nervous system that controls a wide range of physiological events. Accordingly, disruption of DA function has been implicated in the pathogenesis of many psychiatric disorders, including schizophrenia, substance addiction, and attention deficit hyperactivity disorder (ADHD). Since many behaviors and psychiatric conditions show moderate to high heritability, the study of genetic variation in the dopamine-related genes will contribute to unraveling the molecular mechanisms of the catecholamine pathway. These insights into the role of genetic variability could contribute to the development of personalized pharmaceutical intervention based upon the genotype of each individual patient (Housely et al., 2009). DA is involved in the control of locomotion, cognition, affect, and neuroendocrine secretion. These actions of dopamine are mediated by five different receptor subtypes, which are members of the large G-protein coupled receptor superfamily. The dopamine receptor subtypes are divided into two major subclasses: the D1-like (DRD1 and DRD5) and D2-like (DRD2 DRD4) receptors, which are typically coupled to Gs- and Gj-mediated transduction systems (Jaber et al., 1996). All receptors subtypes are expressed in the brain. D1 and D2 are mainly localized to the basal ganglia, while D3, D4 and D5 are found mainly in the cortical and limbic regions. Human DRD5 together with its 5'-flanking and promoter region has been cloned and mapped to chromosome 4p15.1-16.1. DRD5 is structurally similar to DRD1, with both having an overall amino acid homology of 50\%. In addition, DRD5 shows 95\% homology to two pseudogenes that are $98 \%$ homologous to each other (Beischlag et al., 1996). DRD5 binds DA with 10-fold greater affinity compared to DRD1, suggesting that DRD5 may play a more important role in DA synthesis in the brain (Williams et al., 1997). Therefore, DRD5 may also be a candidate gene for schizophrenia. Genetic studies have shown an association between DRD5 and suicidal behavior (Fernández-Navarro et al., 2012), major depression (Nyman et al., 2011), nicotine dependence (Wei et al., 2012), alcoholism (Hack et al., 2011) and ADHD (Housley et al., 2009).

To date, no evidence has been found for a role of DRD5 in schizophrenia, despite several studies in this area (Muir et al., 2001; Ikeda et al., 2008; Pal et al., 2009; Hwang et al., 2012). It is noteworthy that a number of these studies focused mainly on the rs2076907 (-226G/C), a common polymorphic SNP in the 5'UTR (Kim et al., 2007), and rs6283(978T/C), a common polymorphic SNP in the coding region (Sullivan et al., 2001), but there is little evidence that these two SNPs have an association with schizophrenia. There is a dinucleotide microsatellite repeat (CA/ CT/GT)n polymorphism located $18.5 \mathrm{~kb}$ from the $5^{\prime}$ end of DRD5. This polymorphism has been extensively studied in other disorders and found to be associated with ADHD (Li et al., 
2006), a cognitive disorder, as well as cognitive symptoms of schizophrenia (Golimbet et al., 2008) and schizophrenia itself (Williams et al., 1997; Muir et al., 2001).

Past studies suggest that genetic variants of DRD5 could play an important role in the etiology of several psychiatric disorders. However, few association studies of DRD5 with schizophrenia have been conducted, and no positive results have been found to date. Therefore, the aim of the present study was to investigate the association between paranoid schizophrenia in Han Chinese and specific SNPs within DRD5, including rs2076907 and rs6283, which have been previously studied, and two additional SNPs that have received little or no attention to date (rs77434921 and rs1800762).

\section{MATERIAL AND METHODS}

\section{Subjects}

All subjects were ethnically Han Chinese. This case-control study involved 176 unrelated in-patients with paranoid schizophrenia (96 males, 80 females; mean age of $43.0 \pm$ 14.3 years) recruited from the Kaiyuan Mental Hospital in Liaoning Province, China. Patients were diagnosed by at least two psychiatrists according to DSM-IV criteria and CCMD3 for schizophrenia. The control group consisted of 206 unrelated healthy volunteers ( 81 males, 125 females; mean age of $37.5 \pm 15.4$ years) free from present, past, and family history of psychiatric illness. This study was approved by the Medical Research Ethics Committee at China Medical University, and informed consent was obtained from all subjects. A 5-mL peripheral blood sample was obtained from each subject for extraction of genomic DNA.

\section{Genotyping}

Genomic DNA was extracted from leukocytes using a standard phenol-chloroform method. Two fragments that included the four SNPs of interest were amplified from human genomic DNA by PCR. SNPs were genotyped by DNA sequencing analysis using the primers shown in Table 1.

Table 1. Primers used for amplification of the DRD5 gene.
\begin{tabular}{llcc} 
& & \\
\hline Primer & Sequence 5' $\rightarrow$ 3' & Fragment length & SNPs \\
\hline DRD5-1U & TTCGGAGTAGTGAAAGGAGAAGAAGCA & 2163 bp & rs774349921 \\
DRD5-1L & CCTACTCATCATCTGGACCCTGCTG & Rs2076907 \\
DRD5-2U & TGTGCGTCATCAGCGTGGA & 922 bp & rs1800762 \\
DRD5-2L & GGTCCTTTCGATCGCATGTT & & Rs6283 \\
\hline
\end{tabular}

\section{Statistical analysis}

Hardy-Weinberg equilibrium, linkage disequilibrium (LD), and allele-based association analyses of single markers, as well as haplotype analyses, were calculated using Haploview version 4.2. The chi-square test was used to compare genotypic frequencies between schizophrenic patients and controls using the SPSS.14.0 statistical package. All tests were two-tailed, and the level of significance was set at 0.05 . 


\section{RESULTS}

\section{Identification of $D R D 5$ sequence variants}

A 2163-bp 5' promoter region and a 922-bp coding region of the DRD5 gene were sequenced, in addition to four reported SNPs (rs77434921, rs2076907, 0rs6283, and rs1800762) (Table 2).

Table 2. Sequence variants of the human DRD5 gene identified in this current study.

\begin{tabular}{lllc}
\hline Position & dbSNP ID & Region & Allele1/2 \\
\hline-1491 & rs77434921 & 5'flanking region & A/G \\
-226 & rs2076907 & Exon (5'-UTR) & G/C \\
978 & rs6283 & Exon & C/T \\
1004 & rs1800762 & Exon & A/C \\
\hline
\end{tabular}

\section{Single-site association analysis}

Genotype distributions and allele frequencies of the four SNPs in schizophrenic patients and controls are summarized in Table 3. The genotype distributions of the six loci were in Hardy-Weinberg equilibrium for both patients and controls. Chi-square analysis showed significant group differences for distributions of allele $(\mathrm{P}=0.008, \mathrm{OR}=0.442$, $95 \% \mathrm{CI}=0.244-0.799)$ and genotype $(\mathrm{P}=0.011)$ frequencies for $\mathrm{rs} 77434921$. This analysis also showed significant group differences for distributions of allele $(\mathrm{P}=0.006, \mathrm{OR}=$ $0.427,95 \% \mathrm{CI}=0.237-0.771)$ and genotype $(\mathrm{P}=0.004)$ frequencies for $\mathrm{rs} 1800762$. Moreover, when males and females were analyzed separately, there were significant differences in distributions of allele $(\mathrm{P}=0.016, \mathrm{OR}=1.864,95 \% \mathrm{CI}=1.145 \sim 3.036)$ and genotype $(P=0.044)$ frequencies for $r 2076907$ for males but not for females $(P>0.05)$, suggesting that males carrying the $\mathrm{G}$ allele were more susceptible to schizophrenia than females. There were also significant differences in distributions of allele $(\mathrm{P}=0.03, \mathrm{OR}=0.517$, $95 \% \mathrm{CI}=0.334-0.800)$ and genotype $(\mathrm{P}=0.012)$ frequencies for rs6283 for males but not for females $(\mathrm{P}>0.05)$.

Figure 1 shows the data from pairwise LD analyses between schizophrenia and control groups across four SNPs, as represented by the LD coefficient (D'). Strong LD was observed between rs77434921 and rs1800762 in both groups, while strong LD between rs2076907 and rs6283 was present in schizophrenia patients but not in controls, suggesting a possible disease association. Accordingly, we identified two haplotype blocks for further investigation via haplotype association analysis (block 1:rs77434921 and rs1800762; block 2: rs2076907, and rs6283).

\section{Haplotype association analysis}

Table 4 shows the results of the haplotype analysis. Significant group differences were observed for haplotypes $\mathrm{GC}(\mathrm{P}=0.048, \mathrm{OR}=0.466,95 \% \mathrm{CI}=0.215-1.088)$ in block 1. Furthermore, significant group differences in distribution were detected in males ( $P$ $<0.05)$ but not in females $(\mathrm{P}>0.05)$. There were no significant group differences for 


\begin{tabular}{|c|c|c|c|c|c|c|c|}
\hline SNP ID & $\begin{array}{c}\text { Cases } \\
(\mathrm{N}=176)\end{array}$ & No. $\%$ & $\begin{array}{l}\text { Controls } \\
(\mathrm{N}=206)\end{array}$ & No. $\%$ & $\mathrm{P}$ & OR & $95 \% \mathrm{CI}$ \\
\hline \multicolumn{8}{|c|}{ Rs77434921 } \\
\hline \multicolumn{8}{|c|}{ All subjects } \\
\hline GG & 145 & 82.39 & 190 & 92.23 & 0.011 & & \\
\hline $\mathrm{AG}$ & 29 & 16.48 & 14 & 6.80 & & & \\
\hline $\mathrm{AA}$ & 2 & 2.13 & 2 & 0.97 & & & \\
\hline G & 319 & 90.63 & 394 & 95.65 & & & \\
\hline A & 33 & 9.37 & 18 & 4.37 & 0.008 & 0.442 & $0.244-0.799$ \\
\hline \multicolumn{8}{|l|}{ Male } \\
\hline GG & 77 & 80.21 & 76 & 93.83 & 0.028 & & \\
\hline AG & 17 & 17.71 & 4 & 4.94 & & & \\
\hline $\mathrm{AA}$ & 2 & 2.08 & 1 & 1.23 & & & \\
\hline G & 171 & 89.06 & 156 & 96.3 & & & \\
\hline A & 21 & 10.94 & 6 & 3.7 & 0.015 & 0.313 & $0.123-0.796$ \\
\hline \multicolumn{8}{|c|}{ Female } \\
\hline GG & 68 & 85.0 & 114 & 91.2 & 0.215 & & \\
\hline $\mathrm{AG}$ & 12 & 15.0 & 10 & 8.00 & & & \\
\hline AA & 0 & 0 & 1 & 0.8 & & & \\
\hline $\mathrm{G}$ & 158 & 92.5 & 238 & 95.2 & & & \\
\hline A & 12 & 7.5 & 12 & 4.8 & 0.13 & 0.622 & $0.272-1.42$ \\
\hline \multicolumn{8}{|c|}{ Rs2076907 } \\
\hline \multicolumn{8}{|c|}{ All subjects } \\
\hline GG & 89 & 50.57 & 123 & 59.7 & 0.164 & & \\
\hline GC & 69 & 39.20 & 69 & 33.5 & & & \\
\hline $\mathrm{CC}$ & 18 & 10.23 & 14 & 6.8 & & & \\
\hline G & 247 & 70.17 & 315 & 76.46 & & & \\
\hline $\mathrm{C}$ & 105 & 29.83 & 97 & 23.54 & 0.058 & 1.307 & $0.945-1.807$ \\
\hline \multicolumn{8}{|l|}{ Male } \\
\hline GG & 45 & 46.87 & 51 & 62.97 & 0.044 & & \\
\hline GC & 40 & 41.46 & 27 & 33.33 & & & \\
\hline $\mathrm{CC}$ & 11 & 11.46 & 3 & 3.7 & & & \\
\hline G & 130 & 67.71 & 129 & 79.63 & & & \\
\hline $\mathrm{C}$ & 62 & 32.29 & 33 & 20.37 & 0.016 & 1.864 & $1.145-3.036$ \\
\hline \multicolumn{8}{|c|}{ Female } \\
\hline GG & 44 & 55.00 & 72 & 57.6 & 0.924 & & \\
\hline GC & 29 & 36.25 & 42 & 33.6 & & & \\
\hline $\mathrm{CC}$ & 7 & 8.75 & 11 & 8.8 & & & \\
\hline $\mathrm{G}$ & 117 & 73.12 & 196 & 74.4 & & & \\
\hline $\mathrm{C}$ & 43 & 26.88 & 64 & 25.6 & 0.708 & 0.896 & $0.506-1.588$ \\
\hline \multicolumn{8}{|l|}{ Rs6283 } \\
\hline \multicolumn{8}{|c|}{ All subjects } \\
\hline TT & 55 & 31.25 & 70 & 33.98 & 0.603 & & \\
\hline $\mathrm{TC}$ & 88 & 50.0 & 105 & 50.97 & & & \\
\hline $\mathrm{CC}$ & 33 & 18.75 & 31 & 15.05 & & & \\
\hline $\mathrm{T}$ & 198 & 56.25 & 245 & 59.47 & & & \\
\hline $\mathrm{C}$ & 154 & 43.75 & 167 & 40.53 & 0.396 & 0.876 & $0.657-1.169$ \\
\hline \multicolumn{8}{|l|}{ Male } \\
\hline TT & 27 & 28.13 & 39 & 48.15 & 0.012 & & \\
\hline $\mathrm{TC}$ & 49 & 51.04 & 34 & 41.98 & & & \\
\hline $\mathrm{CC}$ & 20 & 20.83 & 8 & 9.87 & & & \\
\hline $\mathrm{T}$ & 103 & 53.65 & 112 & 69.14 & & & \\
\hline $\mathrm{C}$ & 89 & 46.35 & 50 & 30.86 & 0.03 & 0.517 & $0.334-0.800$ \\
\hline \multicolumn{8}{|c|}{ Female } \\
\hline TT & 28 & 35.00 & 31 & 34.8 & 0.289 & & \\
\hline $\mathrm{TC}$ & 39 & 48.75 & 71 & 56.8 & & & \\
\hline $\mathrm{CC}$ & 13 & 16.25 & 23 & 18.4 & & & \\
\hline $\mathrm{T}$ & 95 & 59.38 & 133 & 53.2 & & & \\
\hline
\end{tabular}

Continued on next page 
Table 3. Continued.

\begin{tabular}{|c|c|c|c|c|c|c|c|}
\hline SNP ID & $\begin{array}{c}\text { Cases } \\
(\mathrm{N}=176)\end{array}$ & No. $\%$ & $\begin{array}{l}\text { Controls } \\
(\mathrm{N}=206)\end{array}$ & No. $\%$ & $\mathrm{P}$ & OR & $95 \% \mathrm{CI}$ \\
\hline $\mathrm{C}$ & 65 & 40.62 & 117 & 46.86 & 0.224 & 1.286 & $0.860-1.921$ \\
\hline \multicolumn{8}{|c|}{ Rs 1800762} \\
\hline \multicolumn{8}{|c|}{ All subjects } \\
\hline $\mathrm{CC}$ & 142 & 80.68 & 188 & 91.26 & 0.004 & & \\
\hline $\mathrm{AC}$ & 34 & 19.32 & 18 & 8.74 & & & \\
\hline AA & 0 & 0 & 0 & 0 & & & \\
\hline $\mathrm{C}$ & 318 & 90.34 & 314 & 95.63 & & & \\
\hline $\mathrm{A}$ & 34 & 9.66 & 18 & 4.37 & 0.006 & 0.427 & $0.237-0.771$ \\
\hline \multicolumn{8}{|l|}{ Male } \\
\hline $\mathrm{CC}$ & 75 & 78.13 & 76 & 93.83 & 0.005 & & \\
\hline $\mathrm{AC}$ & 21 & 21.87 & 5 & 6.17 & & & \\
\hline AA & 0 & 0 & 0 & 0 & & & \\
\hline $\mathrm{C}$ & 171 & 89.06 & 157 & 96.91 & & & \\
\hline A & 21 & 10.94 & 5 & 3.09 & 0.007 & 0.259 & $0.095-0.704$ \\
\hline \multicolumn{8}{|l|}{ Female } \\
\hline $\mathrm{CC}$ & 67 & 83.75 & 112 & 89.6 & 0.194 & & \\
\hline $\mathrm{AC}$ & 13 & 16.25 & 13 & 10.4 & & & \\
\hline AA & 0 & 0 & 0 & 0 & & & \\
\hline $\mathrm{C}$ & 147 & 91.88 & 237 & 94.8 & & & \\
\hline A & 13 & 8.12 & 13 & 5.2 & 0.299 & 0.620 & $0.280-1.375$ \\
\hline
\end{tabular}

Data represent N (\%).

haplotype AA in block 1 or for any haplotypes in block 2. Moreover, when males and females were analyzed separately in block 2 , significant group differences in distribution were detected for haplotype $\mathrm{GC}(\mathrm{P}=0.048, \mathrm{OR}=0.466,95 \% \mathrm{CI}=0.215-1.088)$ in males, suggesting that males with haplotype $\mathrm{GC}$ were more susceptible to paranoid schizophrenia than females.

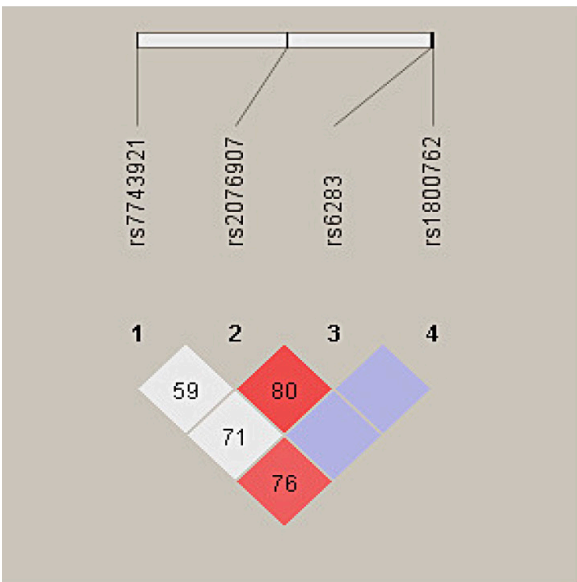

A

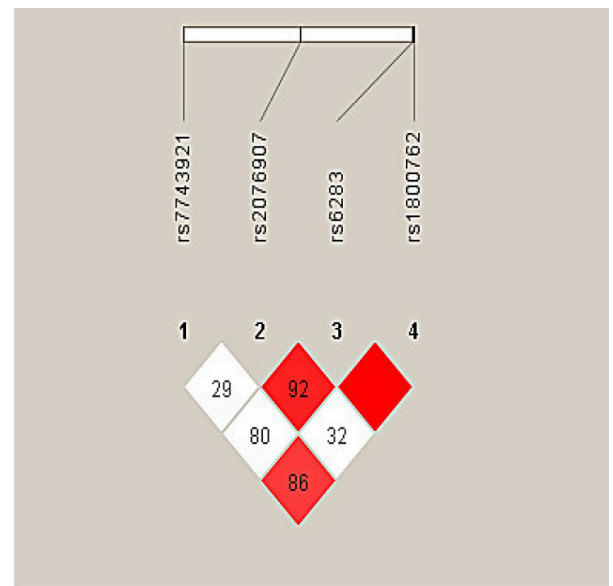

B

Figure 1. Linkage disequilibrium and D' for four SNPs in DRD5 in control subjects (A). and patients with Schizophrenia (B). Empty open diamonds indicate D' $=1$. 


\begin{tabular}{|c|c|c|c|c|c|c|}
\hline Haplotype block & Haplotype $^{a}$ & Cases & Controls & $\mathrm{P}$ & OR & $95 \% \mathrm{CI}$ \\
\hline Block 1: & G-C & & & & & \\
\hline \multirow[t]{7}{*}{ SNPs $1-4$} & All subjects & 0.894 & 0.948 & 0.048 & 0.466 & $0.215-1.088$ \\
\hline & Male & 0.871 & 0.957 & 0.036 & 0.269 & $0.073-0.990$ \\
\hline & Female & 0.918 & 0.940 & 0.383 & 0.619 & $0.209-1.835$ \\
\hline & A-A & & & & & \\
\hline & All subjects & 0.364 & 0.450 & 0.082 & 0.694 & $0.460-1.048$ \\
\hline & Male & 0.374 & 0.425 & 0.544 & 0.829 & $0.453-1.518$ \\
\hline & Female & 0.352 & 0.476 & 0.067 & 0.583 & $0.327-1.040$ \\
\hline Block 2: & CT & & & & & \\
\hline \multirow[t]{11}{*}{ SNPs 2-3 } & All subjects & 0.555 & 0.583 & 0.897 & 1.033 & $0.682-1.564$ \\
\hline & Male & 0.529 & 0.655 & 0.098 & 0.599 & $0.326-1.100$ \\
\hline & Female & 0.580 & 0.511 & 0.378 & 1.290 & $0.733-2.269$ \\
\hline & GC & & & & & \\
\hline & All subjects & 0.280 & 0.205 & 0.088 & 1.507 & $0.939-2.417$ \\
\hline & Male & 0.308 & 0.167 & 0.040 & 2.110 & $1.026-4.340$ \\
\hline & Female & 0.208 & 0.511 & 0.000 & 0.318 & $0.172-0.588$ \\
\hline & $\mathrm{CC}$ & & & & & \\
\hline & All subjects & 0.153 & 0.184 & 0.421 & 0.801 & $0.467-1.375$ \\
\hline & Male & 0.151 & 0.142 & 0.965 & 0.982 & $0.426-2.262$ \\
\hline & Female & 0.154 & 0.225 & 0.192 & 0.611 & $0.291-1.286$ \\
\hline
\end{tabular}

${ }^{a}$ Haplotypes with frequency $<0.01$ were not included in analysis.

\section{DISCUSSION}

To our knowledge, this is the first study to investigate an association between paranoid schizophrenia in Han Chinese and specific SNPs in the DRD5 gene, including rs77434021, rs2076907, rs6283, and rs1800762. These four SNPs were located in both regulatory and coding regions, where the regulatory region includes the promoter region and 5' UTR, which do not alter the protein sequence but may be involved in the regulation of gene expression.

In this study, we successfully identified significant associations in the genotype distributions and allele frequencies of rs 77434921 with paranoid schizophrenia in Han Chinese. Schizophrenic patients had an increased frequency of the A allele, suggesting that the allele maybe confer an increased risk of schizophrenia. The reason may be that rs 77434921 is situated in the promoter region of the DRD5 gene, which impacts expression of DRD5 by modifying binding sites for transcription factors. Moreover, we also identified significant associations in the genotype distributions and allele frequencies of rs 1800762 with paranoid schizophrenia in Han Chinese. Schizophrenic patients had an increased frequency of the A allele, suggesting that the allele may confer an increased risk of schizophrenia. The further analysis revealed that this SNP locus, rs1800762, was a missense mutation of C989-A, resulting in a change in amino acid 330 from a nonpolar, hydrophobic proline to a polar, hydrophilic glutamine. The antheprot protein prediction software was used to show the secondary structure of proteins before and after the mutation, as shown in Figure 2.

Using internal and external membrane scoring, it was found that the mutation at this locus was inside the membrane in the seven-span transmembrane region of the $G$ protein. The receptor membrane structure determines its function and physical and chemical properties and structural changes are likely to affect the correct combination of the corresponding ligand, as shown in Figure 3.

We further evaluated these two significant SNPs to identify at-risk genotypes; however, 
the two significantly associated SNPs showed no significant ORs $(\mathrm{OR}<1)$. Therefore, there was no sufficient evidence of an increased frequency of allele A conferred an increased risk of schizophrenia. The reason for this contradiction may be that the minimum allele frequency (MAF) of these SNPs was small (MAF < 0.05), which leads to a lower statistical power, resulting in a false negative. In addition, when males and females analyzed separately, there were significant differences in distributions of allele $(\mathrm{P}=0.009, \mathrm{OR}=1.726,95 \% \mathrm{CI}=1.144-2.605)$ and genotype ( $P=0.039)$ frequencies for $r s 2076907$ for males but not for females $(P>0.05)$, suggesting that males carrying the $\mathrm{G}$ allele were more susceptible to schizophrenia than females.
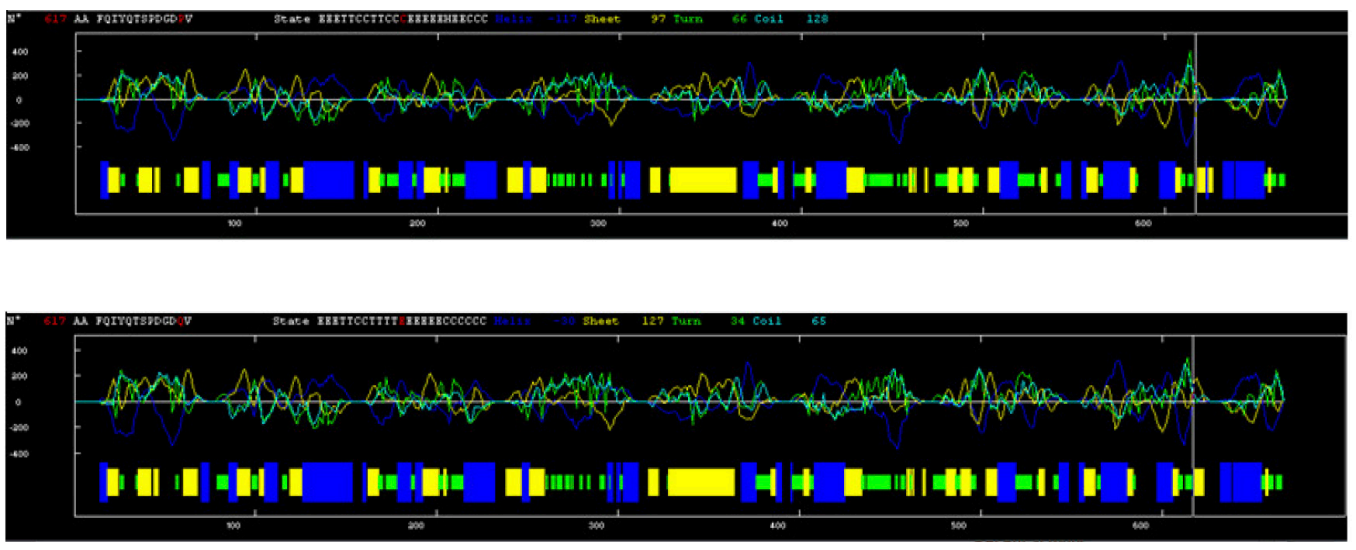

Figure 2. Mimic diagram of the secondary structure of DRD5 encoded proteins.
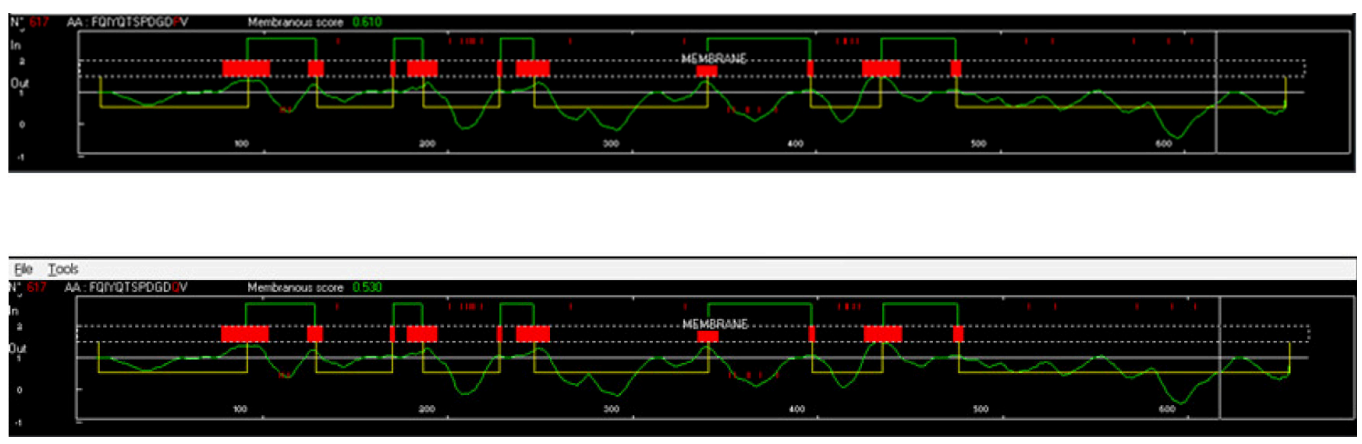

Figure 3. Seven-span transmembrane structure diagram of the DRD5 encoded G protein-coupled receptor.

Moreover, an association was also observed between paranoid schizophrenia in Han Chinese and haplotype frequencies of GC combined with rs77434921-rs1800762. When males and females were analyzed separately, there were significant differences in distributions of GC for males but not for females ( $\mathrm{P}>0.05)$, suggesting that males with haplotype GC were more susceptible to paranoid schizophrenia than females. However, the epidemiological survey showed a significantly higher incidence of mental illness in females. The reasons for this contradiction are unclear and require further study.

In the present study, we found no significant differences in genotypic distributions 
or allelic frequencies of rs6283. Furthermore, frequencies of haplotype AA constructed with rs77434921-rs 1800742 and haplotypes CT and CC constructed with rs2076907-rs6283 were not associated with schizophrenia.

In recent years, a growing number of studies have investigated the relationship between DRD5 and schizophrenia (Muir et al., 2001; Pal et al., 2009; Hwang et al., 2012). Some studies have reported an association between genetic variations in the DRD5 gene and schizophrenia (William et al., 1997; Muir et al., 2001). However, no significant SNPs have been reported in previous studies. The present study was based on a candidate gene approach. It should be noted that, as with other complex diseases, SNPs wide association studies have been initiated to identify genetic risk variants associated with psychiatric disorder. SNPs in the current study were both in the regulatory region and coding region of the DRD5 gene and therefore could affect observed gene expression. Schizophrenia is a heterozygous disease, and the clinical heterogeneity of disease phenotypes may prevent a more detailed understanding of genetic structure (Cherlyn et al., 2010). We specifically studied the paranoid subtype of schizophrenia, and the relatively small sample size of the current study limits the generalizability of our results, so these data should be replicated in an independent study.

In conclusion, we found associations between paranoid schizophrenia in Han Chinese and the genotype distributions and allele frequencies of rs77434921 and rs1800762, as well as haplotype frequencies of GC combined with rs77434921-rs1800762. In addition, the genotype distribution and allele frequency of rs2076907 and haplotype frequencies of GC combined with rs2076907-rs6283 are associated with male paranoid schizophrenia patients. This is the first report to show that specific SNPs in DRD5 are associated with paranoid schizophrenia.

\section{ACKNOWLEDGMENTS}

Special thanks to the Third People's Hospital of Liaoning Province for providing blood samples from paranoid schizophrenic patients for this study.

\section{REFERENCES}

Beischlag TV, Nam D, Ulpian C, Seeman P, et al. (1996). A polymorphic dinucleotide repeat in the human dopamine D5 receptor gene promoter. Neurosci. Lett. 205: 173-176.

Cardno AG and Gottesman II (2000). Twin studies of schizophrenia: from bow-and-arrow concordances to star wars Mx and functional genomics. Am. J. Med. Genet. 97: 12-17.

Cherlyn SY, Woon PS, Liu JJ, Ong WY, et al. (2010). Genetic association studies of glutamate, GABA and related genes in schizophrenia and bipolar disorder: a decade of advance. Neurosci. Biobehav. Rev. 34: 958-977.

Fernández-Navarro P, Vaquero-Lorenzo C, Blasco-Fontecilla H, Diaz-Hernandez M, et al. (2012). Genetic epistasis in female suicide attempters. Prog. Neuropsychopharmacol. Biol. Psychiatry 38: 294-301.

Golimbet VE, Alfimova MV, Gritsenko IK, Lezheiko TV, et al. (2008). Association of dopamine receptor D5 gene polymorphism with peculiarities of voluntary attention in schizophrenic patients and their relatives. Bull. Exp. Biol. Med. 145: 65-67.

Hack LM, Kalsi G, Aliev F, Kuo PH, et al. (2011). Limited associations of dopamine system genes with alcohol dependence and related traits in the Irish Affected Sib Pair Study of Alcohol Dependence (IASPSAD). Alcohol. Clin. Exp. Res. 35: 376-385.

Housley DJ, Nikolas M, Venta PJ, Jernigan KA, et al. (2009). SNP discovery and haplotype analysis in the segmentally duplicated DRD5 coding region. Ann. Hum. Genet. 73: 274-282.

Hwang R, Tiwari AK, Zai CC, Felsky D, et al. (2012). Dopamine D4 and D5 receptor gene variant effects on clozapine response in schizophrenia: replication and exploration. Prog. Neuropsychopharmacol. Biol. Psychiatry 37: 62-75.

Ikeda M, Yamanouchi Y, Kinoshita Y, Kitajima T, et al. (2008). Variants of dopamine and serotonin candidate genes as 
predictors of response to risperidone treatment in first-episode schizophrenia. Pharmacogenomics 9: 1437-1443.

Jaber M, Robinson SW, Missale C and Caron MG (1996). Dopamine receptors and brain function. Neuropharmacology 35: 1503-1519.

Kim DJ, Park BL, Yoon S, Lee HK, et al. (2007). 5' UTR polymorphism of dopamine receptor D1 (DRD1) associated with severity and temperament of alcoholism. Biochem. Biophys. Res. Commun. 357: 1135-1141.

Li D, Sham PC, Owen MJ and He L (2006). Meta-analysis shows significant association between dopamine system genes and attention deficit hyperactivity disorder (ADHD). Hum. Mol. Genet. 15: 2276-2284.

Muir WJ, Thomson ML, McKeon P, Mynett-Johnson L, et al. (2001). Markers close to the dopamine D5 receptor gene (DRD5) show significant association with schizophrenia but not bipolar disorder. Am. J. Med. Genet. 105: 152-158.

Nyman ES, Sulkava S, Soronen P, Miettunen J, et al. (2011). Interaction of early environment, gender and genes of monoamine neurotransmission in the aetiology of depression in a large population-based Finnish birth cohort. BMJ Open 1: e000087.

Pal P, Mihanovic M, Molnar S, Xi H, et al. (2009). Association of tagging single nucleotide polymorphisms on 8 candidate genes in dopaminergic pathway with schizophrenia in Croatian population. Croat. Med. J. 50:361-369.

Schwab SG and Wildenauer DB (2008). Research on causes for schizophrenia: are we close? Schizophr. Res. 102: 29-30.

Sullivan PF, Neale MC, Silverman MA, Harris-Kerr C, et al. (2001). An association study of DRD5 with smoking initiation and progression to nicotine dependence. Am. J. Med. Genet. 105: 259-265.

Wei J, Chu C, Wang Y, Yang Y, et al. (2012). Association study of 45 candidate genes in nicotine dependence in Han Chinese. Addict. Behav. 37: 622-626.

Williams NM, Cardno AG, Murphy KC, Jones LA, et al. (1997). Association between schizophrenia and a microsatellite polymorphism at the dopamine D5 receptor gene. Psychiatr. Genet. 7: 83-85. 